\title{
La familia en la estimulación del hábito lector en niños de cuatro a seis años
}
The family in the stimulation of the reading habit in four-six year old children

\section{EVELYN SAGAL*(D), VERÓNICA CARVAJAL (a) (D) Y MARÍA DEL ROSARIO REQUENA ${ }^{(b)}(\mathbb{D})$}

(a) Universidad de las Fuerzas Armadas ESPE,

Av. General Rumiñahui y Ambato, 170501. Sangolquí - Ecuador,

(b) Universidad Técnica Particular de Loja, Loja- Ecuador,

San Cayetano Alto, Calle París, Loja - Ecuador

*easagal@espe.edu.ec

\section{RESUMEN}

El hábito lector es un proceso que se debe estimular en niños desde su primera infancia y los padres de familia son los responsables de la motivación de la lectura en el hogar. En nuestro país existen limitados estudios sobre los hábitos de lectura, sumando a ello las pocas investigaciones que se registran acerca de las estrategias que utilizan los padres para animar a esta importante actividad con sus hijos de cuatro a seis años. El trabajo investigativo realizado es de tipo cuantitativo-descriptivo, mediante la aplicación de una encuesta a padres de familia que tienen niños de cuatro a seis años. Este trabajo se desarrolló en la ciudad de Quito-Ecuador y permitió investigar aspectos como: prácticas o hábitos lectores de la familia, valoración del papel de la lectura, tipo de material de lectura utilizada en el hogar, las estrategias y actividades lectoras que los padres emplean con sus hijos. En conclusión, los padres presentan escasas prácticas lectoras y conocimientos de cómo estimular la acción de leer en sus hijos.

Palabras clave: Familia, lectura, hábitos, estimulación, estrategias. 


\section{ABSTRACT:}

The reading habit is a process that should be stimulated in children from early childhood and parents are responsible for the motivation to read at home. In our country, there are limited studies on reading habits, adding to this the few investigations that are registered about the strategies that parents use to encourage their four-six year old children to develop this important reading habit. This research is quantitative-descriptive through the application of a survey to parents having children between four and six years old. This work was developed in the city of QuitoEcuador and allowed to investigate aspects such as: reading practices or habits of the family. It also helped assess the role of reading, the type of reading material used at home, the reading strategies, and the activities that parents use with their children. In conclusion, parents have little knowledge of how to stimulate their children' reading skill.

Keywords: Family, reading, habits, stimulation, strategies.

\section{INTRODUCCIÓN}

El hábito lector es uno de los procesos más complejos al que se le dedica mucho tiempo y esfuerzo, especialmente en edades tempranas. Su enseñanza se realiza para asegurar la interacción y adaptación cognitiva y social de una persona al mundo, particularmente, para facilitar la adquisición de conocimientos y saberes (Vasylenko, 2020), que serán aplicados a un sinfín de actividades como: comunicarse con otros, entender e interpretar correctamente lo que dice un texto, etc. (Ramírez \& De Castro,
2013). Dicho proceso no solo implica a las instituciones educativas, sino también al contexto familiar. Es un hecho que el desarrollo de la costumbre lectora tenga que ver más con el factor hereditario que con la escuela (Paredes M., 2014). Esto nos da a entender que los niños y las niñas aprenden a leer por medio de sus relaciones sociales más cercanas: la familia, quienes desempeñan funciones importantes en el inicio del interés por la lectura de sus hijos (Ortiz, 2017). Resulta difícil que un niño logre ser lector si no se crea un vínculo afectivo con los libros desde una temprana edad (Rosero \& Mieles, 2015).

De ahí la importancia de comprometer a las familias a estimular el proceso de aprendizaje lector de sus hijos para que ellos desarrollen de manera activa la motivación y el deleite por la lectura (Kalb \& Ours, 2020). Por ende, este hábito debe desarrollarse a partir de diferentes espacios y experiencias que brinden oportunidades enriquecedoras que incentiven un acercamiento afectivo al mundo de la lectura (Matvichuk, 2020).

En el contexto internacional, la Unesco (Organización de las Naciones Unidas para la Educación, la Ciencia y la Cultura) considera a la lectura como una puerta que ayuda a comprender e interpretar el mundo, y los libros permiten al lector tener una mente más abierta y tolerante, aunque en algunos países sigue siendo un reto adquirir la habilidad lectora (Unesco, 2016). Para el programa PISA (Program for International Student Assessment), la lectura es esencial para que los individuos puedan realizar actividades cotidianas: desde seguir las instrucciones de un manual, hasta averiguar el quién, cuándo, qué, dónde y porqué de un evento y comunicarse con sus semejantes. En su informe, las ciudades como: Beijing, Shanghai, Jiangsu y Zhejiang (China) 
y Singapur obtuvieron una puntuación significativamente más alta en lectura que el resto de los países/economías participantes en PISA 2018. Estonia, Canadá, Finlandia e Irlanda fueron los países de la OCDE con el rendimiento más alto en lectura. Sin duda, detrás de estos resultados se evidencia un arduo trabajo de las familias, la escuela y el Estado, para fomentar el hábito de lectura, lo cual se refleja en los resultados de sus estudiantes.

\subsection{La importancia de la estimulación para la adquisición de la lectura}

En lo que respecta a la adquisición del hábito lector, los progenitores tienen que tomar en cuenta que formar hábitos de lectura es de suma importancia, debido a que deben ser instaurados siempre de acuerdo al grado de madurez que posee el niño o la niña y jamás intentar sobre exigirles, puesto que lo único que se logrará es generar desinterés en ellos. La lectura no debe ser impuesta, esta debe ser motivada por medio del juego y basada en el interés del niño (Roque \& Álvarez, 2016).

Dentro de las experiencias más significativas en la formación de hábitos lectores en los niños, está el observar a sus progenitores leyendo; por este motivo la labor de estimular la lectura requiere de un arduo trabajo y dedicación de la familia, la cual tiene el papel de orientar la formación de hábitos en sus hijos, tal como lo demuestra Díaz y Vargas (2015) en su investigación "Aproximación temprana a la lectura: el caso de Federico", en la que se muestra a un niño que recibió estimulación lectora por parte de sus padres desde los 10 meses de nacido, como resultado, el infante muestra destreza en la manipulación de libros, presenta imitación de comportamientos lectores e incremento su vocabulario.

Asimismo, un estudio realizado por Bradley y Bryant (1983), uno de los pioneros en problemas infantiles de lectura, demostró que muchos de los niños examinados con dificultades lectoras presentaban una escasa estimulación en los sonidos de la lengua dentro de su contexto familiar, los cuales son requisitos fundamentales para evitar el fracaso en el proceso de aprendizaje de la lectura; también existe una teoría principal que aborda la estimulación como aspecto importante para favorecer la usanza de la lectura en los niños: la teoría psicolingüística.

\section{La teoría psicolingüística}

Uno de los principales autores que defienden esta teoría son el lingüista Noam Chomsky; los psicólogos Jean Piaget y el ruso Lev Vygotsky, entre otros. Esta teoría, según Suárez (2013), refiere que el niño es capaz de concebir lo que lee mediante dos clases de habilidades cognitivas: reconocimiento y acceso al significado de las palabras, y las acciones mentales que contribuyen en la interpretación del texto. Por consiguiente, los niños que crecen en contextos letrados aprenden con mayor facilidad a identificar las palabras, dibujos u otros símbolos (Sepúlveda \& Gaintza 2017).

Sin lugar a dudas, la estimulación que los padres dan a sus hijos antes de que ellos aprendan a leer es de gran ayuda para ir desarrollando este hábito y evitar en un futuro complicaciones que tengan que ver con la falta de fluidez en palabras más complejas o una lectura lenta y silábica. 


\subsection{Hábito de lectura en Ecuador}

Las pocas investigaciones realizadas en nuestro contexto afirman que el hábito de la lectura es una de las más grandes deficiencias culturales del país. Así lo menciona en su último estudio el Instituto Nacional de Estadística y Censos INEC (2012), el cual obtuvo como resultados que el $27 \%$ de los ecuatorianos no tienen la práctica de la lectura. Para el INEC, la razón principal es la falta de interés que corresponde al 56,8 \%. Esto es debido a que el 50,3 \% leen entre una y dos horas semanales. El $33 \%$ de los jóvenes que leen lo hacen para atender obligaciones académicas, más no por placer. En lo que respecta a lectura de libros, la Cámara Ecuatoriana del Libro determinó que el índice de lectura del ecuatoriano es de 0,5 libros por año, en otras palabras, cada persona lee medio libro al año (Andes, 2013), ubicándolo lejos de Chile y Argentina que registran un 5,4 y 4,6, México y Colombia, se ubican por debajo con 2,9 y 2,2 libros leídos al año por habitante (Cerlalc, 2012). En lo que respecta a España, leen 13 libros y Finlandia 47 libros al año (Unesco, 2016).

Frente a estos acontecimientos, nuestro país ha venido trabajando en estrategias con el objetivo de favorecer el hábito lector de sus estudiantes, por lo cual ha realizado los siguientes eventos y programas: el Día del Libro, el seminario "Hacia la construcción de un Plan Nacional de Lectura" organizado por la Asociación Ecuatoriana del Libro Infantil y Juvenil (Girándula), en Quito se realiza el Maratón del Cuento para incentivar la lectura en los niños y la Feria del Libro. En enero 2016 el Ministerio de Educación anunció un "Plan Lector" y dentro de este, un Club de Maestros Lectores (García, 2016).
Dentro de las investigaciones realizadas y que han sido consultadas, se evidencia estudios relacionados con los hábitos de lectura en la población general ecuatoriana y como están realizando eventos para estimularlo, sin embargo, no se evidencian investigaciones que hayan abordado el estudio de los hábitos de lectura y las estrategias utilizadas por los padres para desarrollar el hábito de la lectura en los niños de cuatro a seis años. Dado que, el INEC, solo arroja datos provenientes de 3.960 hogares y toma como población de estudio a jóvenes de 16 años en adelante; pero no identifica el grupo que corresponde a padres de familia y sus conocimientos acerca de la estimulación lectora de sus hijos.

Es así como surgen las interrogantes acerca de: ¿̇Qué prácticas lectoras tienen los padres de familia? ¿Qué tipo de material de literatura infantil existe en los hogares? ¿Qué importancia le dan a la lectura en casa? ¿̇ué estrategias son utilizadas por los padres para introducir al niño en la lectura? De acuerdo a estas preguntas, se plantea el objetivo de conocer las prácticas lectoras y estrategias que realizan los padres de familia en sus hogares con el propósito de estimular la práctica de la lectura en niños.

\subsection{Lectura y familia. Evidencias científicas}

Existen estudios que respaldan el importante papel que cumple la familia respecto al hábito y el gusto por leer; dicha importancia se refleja en la existencia de material destinado para esta práctica, el tiempo que dedican a la lectura, estrategias y actividades que los padres realizan con sus hijos dentro del hogar. Según Ferreiro (2000), los niños y las niñas que han tenido contacto con lectores, 
antes de aprender a leer, adquieren este hábito más fácilmente que aquellos quienes no han tenido este contacto. Igualmente, la Consejería de Educación Junta de Andalucía (2017) realizó un análisis a las pruebas PISA (Program for International Student Assessment), donde la puntuación media obtenida por los alumnos en comprensión lectora se debe a la presencia de libros y el tiempo dedicado a la lectura en el hogar.

De igual manera, el informe PIRLS (Progress in International Reading Literacy Study), son pruebas efectuadas a alumnos de $4^{\circ}$ de Educación Primaria (niños de 8-9 años) para medir la comprensión lectora. Al igual que el informe PISA, llegan a la conclusión de que, si los padres de familia presentan interés y apoyan la lectura, sus hijos tienen como resultado una mejor comprensión lectora (Howie, 2017).

También, este tipo de evidencia afirma que la existencia de libros en los hogares y el gusto e interés por la lectura que los progenitores demuestran en su hogar son factores determinantes para generar este hábito; por ende, el contexto tanto familiar como social, donde crecen los niños y las niñas, influye de modo sobresaliente en el campo de la lectura.

Lo expuesto concuerda con el postulado de la teoría Vigostkiana, (1978) al considerar el desarrollo del aprendizaje, íntimamente relacionado con las oportunidades que tengan los niños y las niñas dentro de su entorno; también se relaciona con lo expuesto por Bronfenbrenner (1987) y Bruner (1977), quienes destacan el papel activo y el rol crucial que desempeñan los adultos en el aprendizaje, puesto que son sujetos a imitar e impulsores del diálogo permanente en familia respecto a la lectura de los infantes.
Por otra parte, el Ministerio de Educación de Colombia (2016) afirma que los niños y las niñas que se desarrollan en un hogar donde los padres muestran apoyo y estimulan este hábito a través de canciones, narración de cuentos, juegos de palabras, y donde se lee en voz alta a los niños desde pequeños, provoca muchos beneficios como expresarse correctamente, prosperando en el aprendizaje formal de la lectura con mayor facilidad.

De igual forma, el Ministerio de Educación de Ecuador (2019) reconoce el derecho y la responsabilidad de la participación de los padres de familia en el aprendizaje de sus hijos, dado que ellos son los primeros gestores que deben estimular y crear un ambiente lector favorable dentro del hogar, con el objetivo de desarrollar competencias lectoras indispensables para aprender nuevos conocimientos.

\subsection{Estrategias para estimular el hábito de la lectura en el hogar}

Un hogar que ofrece oportunidades a sus hijos de interactuar con diferentes tipos de textos, antes de la escolarización, ayuda a potencializar los procesos de pensamiento de los niños (Baker, 2020). Es por eso que los padres de familia deben conocer el desarrollo de los niños, en especial a la edad de 4 a 6 años, porque los infantes en esta etapa son muy curiosos y preguntan el porqué de las cosas; por lo general puede iniciarse por medio de canciones tradicionales o de cuna donde internalizan diferentes tonalidades de voz, ritmos, melodías y expresiones, lo cual permite trabajar la prelectura y el afianzamiento de los lazos afectivos en la familia (Banihashemi, 2011). 
El material con el que pueden optar para estimular la lectura son carteles, fotografías, revistas, periódicos, cuentos, fábulas, poesías, adivinanzas, retahílas, rondas, canciones tradicionales e infantiles, entre otros. Estos son algunos materiales que pueden ser utilizados por los padres de familia para estimular el hábito de la lectura en los niños de cuatro a seis años; pero hay que tomar en cuenta en dónde se adquiere este tipo de material, en especial los textos de lectura, así lo afirma Escalante \& Caldera (2008). Todo material referente a literatura infantil debe ser adquirido en un lugar certificado, como librerías o ferias de libro, dado que estos espacios brindan textos según las características lectoras que requiere el niño según su edad (Chávez, 2015).

Es de suma importancia que los padres tengan conocimiento de los procedimientos estratégicos para trabajar la lectura con sus hijos, por ejemplo, pueden proponer actividades como: realizar predicciones a partir de la referencia previa del texto, de las características de este, de los encabezados o ilustraciones o del conocimiento previo que se posea del tema; realizar anticipaciones tomando como referente el contexto, crear un espacio destinado solo para la lectura, hablar sobre los personajes de los cuentos, observar láminas para hacer predicciones acerca de lo que puede ocurrir, leer en voz alta con una debida entonación, representar los personajes de historias mediante dramatizaciones, imaginar el desenlace y personajes de una historia, inventar finales alternativos, cambiar de rumbo a las historias, imaginar los significados desconocidos y hacer sus propias historias o cuentos (Rendón, 2018).

De la misma forma, durante la evolución del niño, el tiempo dedicado a las actividades relacionadas con la alfabetización es sustancial para la adquisición de las destrezas lectoras, dado que cada infante posee particularidades diferentes, propias de su desarrollo evolutivo, por lo que a esta edad el tiempo apropiado para estimular es de 20 a 25 minutos, con un respectivo descanso para pasar a otra actividad, caso contrario, se llegaría a fatigar y perdería toda la atención (Carrión, 2017).

Evidentemente, es fundamental que los progenitores tengan conocimiento de estas estrategias, dado que no solo generan el gusto por la lectura, sino también, permiten el desarrollo afectivo y psicológico de los pequeños, mejora su atención, concentración y capacidad de escucha, les permite reflexionar sobre el contenido y aumenta su vocabulario (Maluf \& Sargiani, 2013). A su vez, este hábito puede brindar diferentes experiencias y sensaciones, desarrollando de esta manera su imaginación y creatividad. Además, estimula la actividad cerebral y fortalece las conexiones cerebrales, lo cual permite que los niños y las niñas estén activos para cualquier actividad que ejecuten en su labor cotidiana (Mata, 2016).

\section{METODOLOGÍA}

\subsection{Diseño}

El método empleado para esta investigación es cualitativo y responde a un enfoque de tipo descriptivo. Según Hernández, Fernández y Baptista (2018) "Este tipo de investigación busca especificar propiedades y características importantes de cualquier fenómeno que se analice" (p. 64), con este enfoque se buscó conocer las estrategias y procedimientos que los padres realizan para estimular el hábito lector en sus hijos. 


\subsection{Participantes}

En este estudio participaron 65 padres de familia de niños con edades comprendidas entre los cuatro a seis años, pertenecientes al barrio "San Francisco de Huarcay", ubicado al sur de Quito. Del total de participantes $(n=65)$; el número de padres de niños de 4 años $(n=33)$, y padres de niños de 5 a 6 años $(n=32)$.
Como criterio de inclusión, para ser participante de la investigación se tomó en cuenta a los padres de familia de niños que tuvieran la edad de cuatro a seis años. A continuación, se dan a conocer las características sociodemográficas de los padres de familia.

Tabla 1

Características sociodemográficas de la muestra participante

\begin{tabular}{|c|c|c|c|}
\hline & $\begin{array}{l}\text { Padres de niños } \\
\text { de } 4 \text { años }\end{array}$ & $\begin{array}{l}\text { Padres de niños de } \\
\text { cinco a seis años }\end{array}$ & Total \\
\hline \multicolumn{4}{|l|}{ Género } \\
\hline Hombre & 9 & 15 & 24 \\
\hline Mujer & 24 & 17 & 41 \\
\hline Total & 33 & 32 & 65 \\
\hline \multicolumn{4}{|l|}{ Edad } \\
\hline 20-25 años & 16 & 15 & 31 \\
\hline $25-30$ años & 6 & 5 & 11 \\
\hline 30-35 años & 8 & 6 & 14 \\
\hline 40-45 años & 1 & 3 & 4 \\
\hline Más de 50 años & 2 & 3 & 5 \\
\hline Total & 33 & 32 & 65 \\
\hline \multicolumn{4}{|l|}{ Formación académica } \\
\hline Primaria & 8 & 9 & 17 \\
\hline $\begin{array}{l}\text { Cursos técnicos } \\
\text { (peluquería, corte y } \\
\text { confección, etc.) }\end{array}$ & 9 & 7 & 16 \\
\hline Secundaria & 11 & 10 & 21 \\
\hline Superior (Universidad) & 5 & 6 & 11 \\
\hline Total & 33 & 32 & 65 \\
\hline \multicolumn{4}{|c|}{ Número de miembros de la familia } \\
\hline 3 a 4 miembros & 13 & 19 & 32 \\
\hline 5 a 6 miembros & 15 & 11 & 26 \\
\hline Más de 7 miembros & 5 & 2 & 7 \\
\hline Total & 33 & 32 & 65 \\
\hline
\end{tabular}

Nota. Características socio demográficas de los 65 padres de familia, de ambas edades. 


\subsection{Tipo de muestra}

Para esta investigación se aplicó un muestreo no probabilístico. Para (Nádas \& Rakovics, 2017): "Este muestreo permite obtener resultados de una manera más rápida y sencilla en comparación del probabilístico, dado que el investigador conoce la muestra y los participantes comúnmente expresan sus respuestas rápidamente en comparación con personas que se seleccionan al $\operatorname{azar}^{\prime \prime}$ (p. 23).

Este muestreo se aplicó debido al aislamiento social producido por la pandemia del Covid-19 que se vive hoy en día, lo cual dificulta la recogida de información presencial. Es por esta razón que se tomó esta muestra por conveniencia, debido a factores como: cercanía geográfica y contactos de miembros que conforman la organización en el sector a investigar (Osorio, 2019).

\subsection{Instrumento de recogida de información}

Para llevar a cabo este estudio se empleó una encuesta on-line. Para Lorca (2016) "Son una herramienta que los investigadores utilizan para recolectar información a través de una serie de preguntas utilizando la Internet como medio de difusión" (p. 14).

Mediante la revisión de la literatura se pudo identificar cuatro categorías necesarias para analizar la estimulación lectora de las familias encuestadas, tomando en cuenta los estudios de los autores Yusof (2020), Matvichuk (2020), Moreno (2001). Dichas categorías son las siguientes: a) prácticas o hábitos lectores de la de lectura utilizada en el hogar, y d) las estrategias y actividades que los padres realizan para estimular este hábito.

Estas categorías se utilizaron para diseñar una encuesta conformada por 19 preguntas de base estructurada, dirigida a padres de familia.

\section{Confiabilidad y validez (Alfa de Cronbach)}

Es necesario evaluar la confiabilidad y validez del instrumento de medición, solo a través de ello se verificará que la investigación sea seria y aporte resultados reales. Para demostrar la validez del instrumento de esta investigación se realizó una prueba piloto a 70 familias. Como resultado, se efectuó la corrección de dos preguntas correspondientes a las actividades y estrategias que estimulan la lectura por haber sido poco entendibles para los participantes en la prueba. Aparte de estas preguntas, no se registraron otros problemas relacionados con la comprensión del cuestionario en la aplicación de la prueba piloto, la cual fue realizada vía online.

Para poder estudiar la confiabilidad del instrumento utilizado se aplicó la prueba Alfa de Cronbach, que relaciona las variables y establece la confiabilidad de cada sección del cuestionario, el cual tuvo un coeficiente de 0,858 , lo que muestra un nivel de confiabilidad aceptado. Para (Milton, 2010), este coeficiente debe oscilar entre 0 a 1 , el cual debe acercarse a uno para tener mayor confiabilidad, si dicho resultado es menor a 0,7 se tiene el riesgo de que el cuestionario no sea confiable. 
La versión final de la encuesta constó de 2 preguntas abiertas y 17 preguntas de opción múltiple con única respuesta.

\subsection{Procedimientos}

Una vez obtenida la aprobación del Comité central del barrio "San Francisco de Huarcay" para la ejecución de la investigación, se procedió a seleccionar y contactar a los padres de familia, por medio de correo electrónico, en el cual se envió redactada la explicación, propósito, objetivo y procedimiento del presente estudio. Dicha información fue obtenida a través de una matriz de datos de las familias que fue facilitada por el mismo comité.

Posteriormente, los padres de familia participantes respondieron una encuesta online, la cual analizaba los conocimientos de los progenitores acerca de la estimulación del hábito lector que realizan con sus hijos dentro del hogar.

\subsection{Análisis de datos}

El análisis de la información describe los datos obtenidos de la encuesta. A partir de ellos, se realizó el respectivo análisis estadístico que permitió relacionar sus variables. Es decir, se utilizó la estadística descriptiva para cada variable con el fin de describir la relación entre ellas (Hernández, Fernández, Baptista, 2018).

Con los datos recogidos a través de la encuesta de Google Forms, se procedió a descargar la hoja de cálculo de Microsoft Excel, mediante esto se realizó el análisis estadístico descriptivo. Dichos resultados fueron importados al programa estadístico SPSS (Statis- tical Package for the Science), los cuales fueron empleados para construir una base de datos de frecuencias.

En lo que respecta a las preguntas abiertas, para su debido análisis se les asignó un número para poder manejarlas cuantitativamente, conforme su frecuencia de aparición.

\section{RESULTADOS}

Para facilitar la comprensión de los datos recogidos a través de la encuesta se presentarán de acuerdo a cuatro apartados: prácticas de lectura de la familia, valoración del papel de la lectura, tipo de material de literatura infantil, actividades y estrategias que emplean para estimular el hábito lector.

Con respecto al primer apartado de la encuesta que buscaba rastrear las costumbres de la lectura que los padres tienen, se encontró que 29 de los 65 encuestados, que representa el $46 \%$, disfrutan leer revistas sociales (farándula, vanidades, hogar); 18 (27\%) leen en sitios web; 8 (10\%) prefiere leer el periódico; 2 (3 \%) leen novelas, la Biblia, libros de ciencia ficción y superación personal. Los cómics y la poesía presentan poca aceptación, tan solo el $1 \%$ lo prefiere (Figura 1).

Curiosamente, de los 65 encuestados, 34 padres de familia de ambos niveles, que representan el $53 \%$, afirman emplear la lectura como uso exclusivo para la realización de tareas escolares; 6 (9\%) lo emplean como entrenamiento para adultos, y tan solo el $38 \%$, dicen compartir la lectura como medio de entretenimiento para sus hijos (Figura 1). 


\section{Figura 1}

Gustos y empleo de lectura

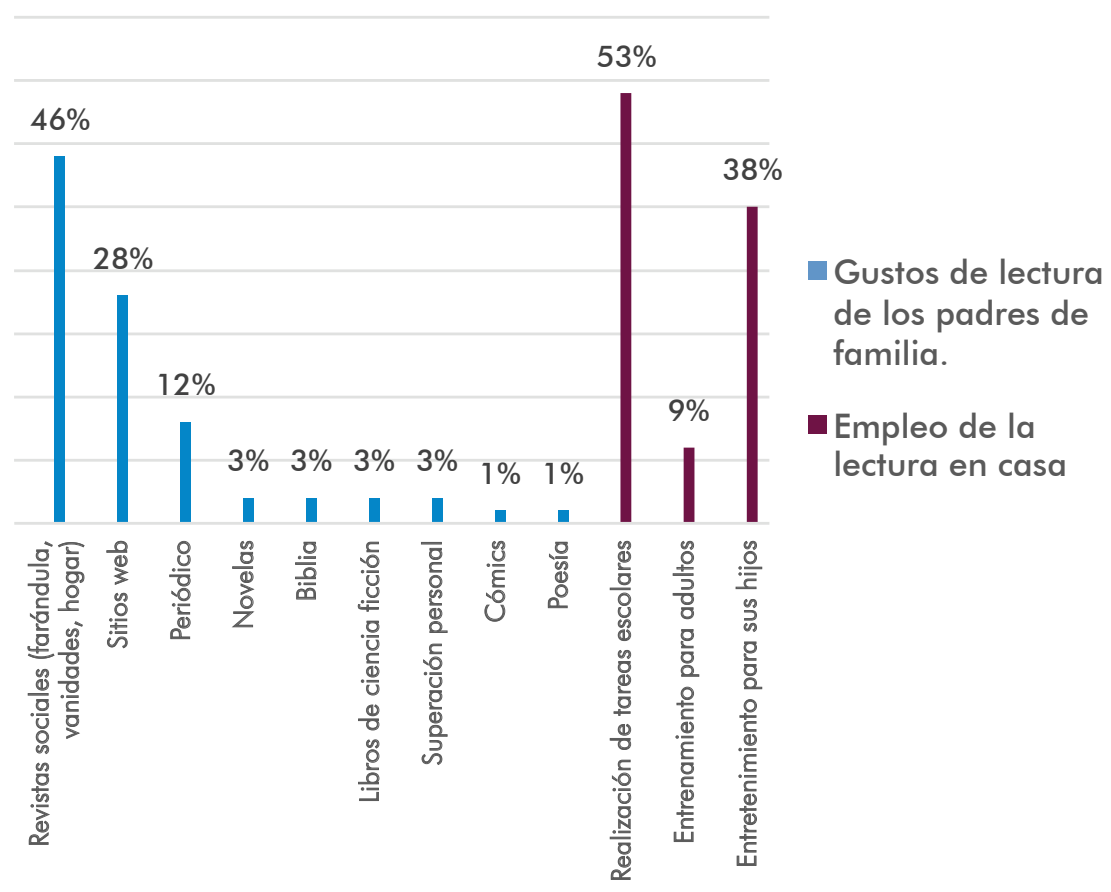

Nota. Resultados de la encuesta prácticas de lectura de la familia

Ahora bien, ambos grupos (50 padres de los 65 encuestados, que representan el $76 \%$ ) indican emplear como principal fuente de acceso a información de lectura, por medio de Internet, siendo la frecuencia de lectura en formato digital la más aplicada.

En lo que respecta al segundo apartado de la encuesta, la valoración que tienen los padres a la lectura, se encontró que la mayoría de los encuestados (54 padres de los 65 encuestados, es decir, $77 \%$ ) apoyan la lectura y el $23 \%$ no lo hace (Figura 2). Por otro lado, la importancia que le dan a la estimulación de esta refleja también un porcentaje alto en los dos grupos (52 padres, que representan el $80 \%$ ) consideran muy importante la estimulación de la lectura, $14 \%$ poco y $6 \%$ nada (Figura 2).

\section{Figura 2}

Apoyo e importancia de la estimulaciòn de la lectura en el hogar

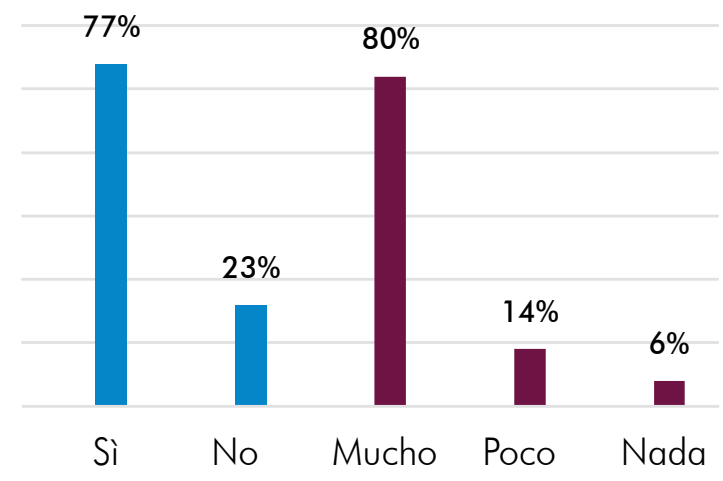

- Apoyo a la lectura

- Importancia de la estimulación de la lectura en los niños

Nota. Resultados de la encuesta valoración del papel de la lectura

Por otra parte, el tiempo que dedican a la lectura (56 padres de los 65 encuestados, que representan el $86 \%$ ), afirman leer de 5 a 10 minutos al día; 6 (9\%) 30 minutos; 2 (3\%) una hora, y el $2 \%$ asegura leer más de una hora (Figura 2). Asimismo, 43 padres, que representan el $66 \%$, aseguran que la tarde es la franja horaria destinada para leer con sus hijos, 16 (25\%) en la mañana y $6(9 \%)$ revelan que leen en la noche (Figura 3). 
Figura 3

Tiempo y franja horaria destinada a la lectura

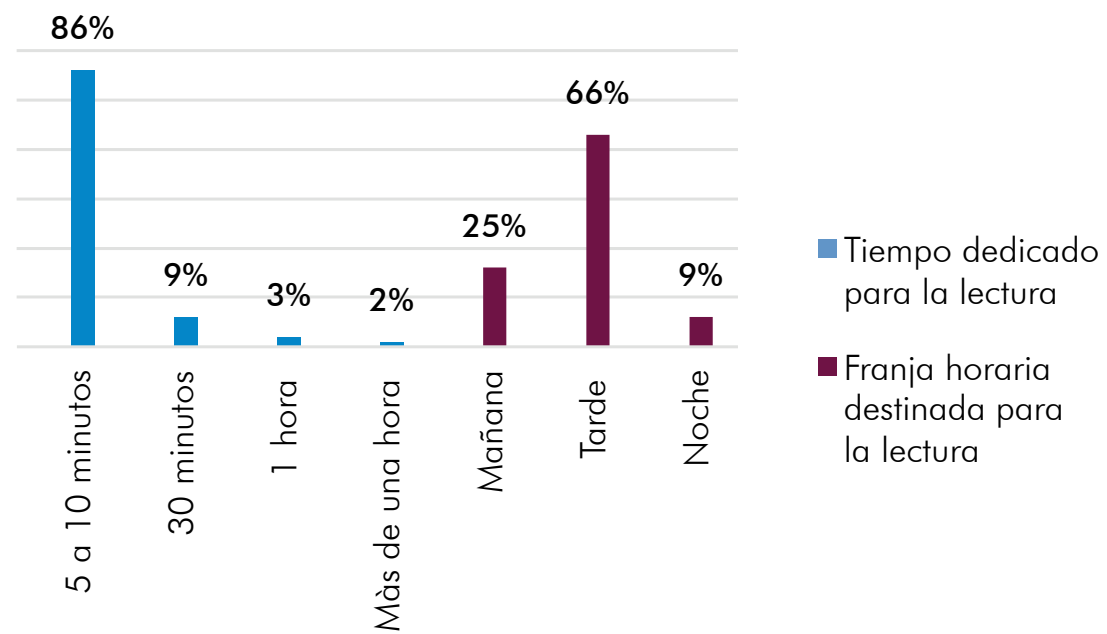

Nota. Resultados de la encuesta valoración del papel de la lectura

En lo que respecta a la existencia de un lugar para la lectura en casa, se halló que la mayoría de los encuestados (4 1 padres de 65 encuestados, es decir, $63 \%$ ), no tienen un lugar establecido para la lectura, solo 14 padres (22\%) aseguran tener un espacio en el cuarto, 8 (12\%) tienen una la sala y $2(3 \%)$ aseguran tener implementada una biblioteca en casa.

En cuanto a la existencia de materiales que motiven la lectura de los niños, se encontró que 34 padres de familia, que corresponde al $52 \%$ indican que poseen cuentos; 13 (20\%) manifiestan tener revistas infantiles (La pandilla, Veo veo, Lee, etc.); 7 (10\%) afirman tener canciones infantiles; 4 (6\%) aseguran tener poesías, $3(5 \%)$ poemas, y 2 (3\%) tienen fábulas.

\section{Figura 4}

Materiales y espacio de lectura

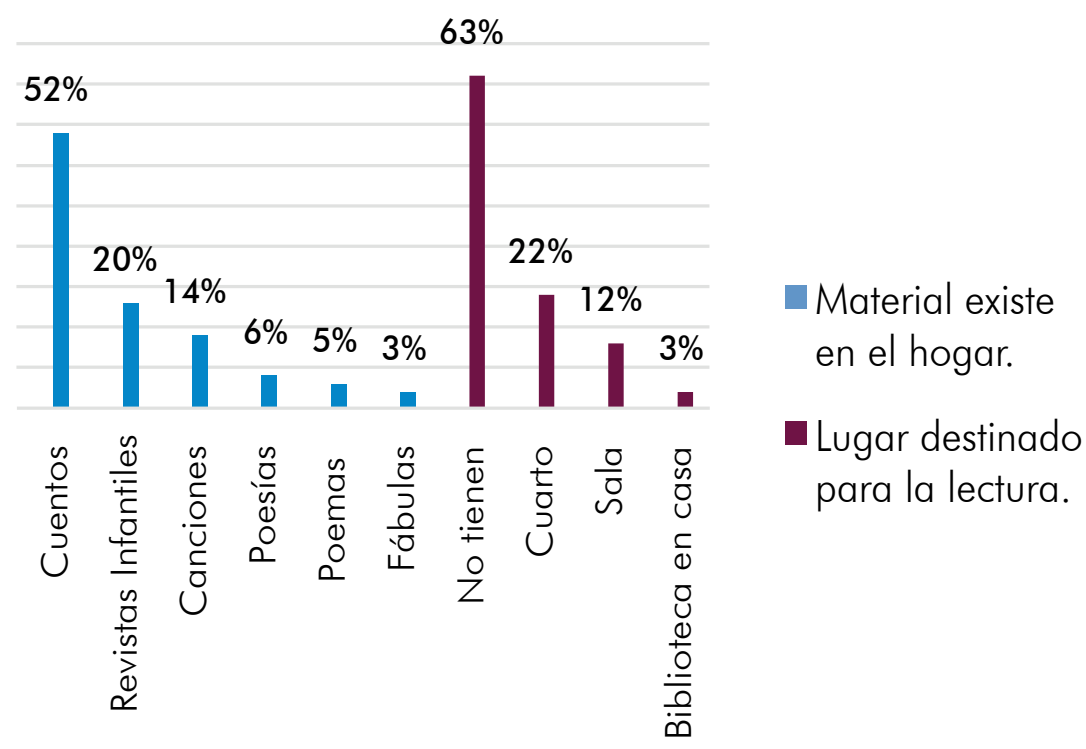

Nota. Resultados de la encuesta tipo de material de literatura infantil

En lo que respecta a la frecuencia con la que compran este tipo de material (42 padres, de los 65 encuestados, es decir, $65 \%)$ admiten a veces comprar material de lectura; 15 (23 $\%)$ lo hacen regularmente, y 8 (12\%) nunca lo hacen. Asimismo, la mayor parte de los progenitores (30 padres de los 65 encuestados, el $46 \%$ ) acostumbran a comprar este tipo de material a vendedores ambulantes, 20 (31 \%) en puestos de revistas, $12(18 \%)$ en librerías y $3(5 \%)$ en ferias de libros (Figura 5). 


\section{Figura 5}

Frecuencia y lugar donde compran material de lectura

\section{$65 \%$}

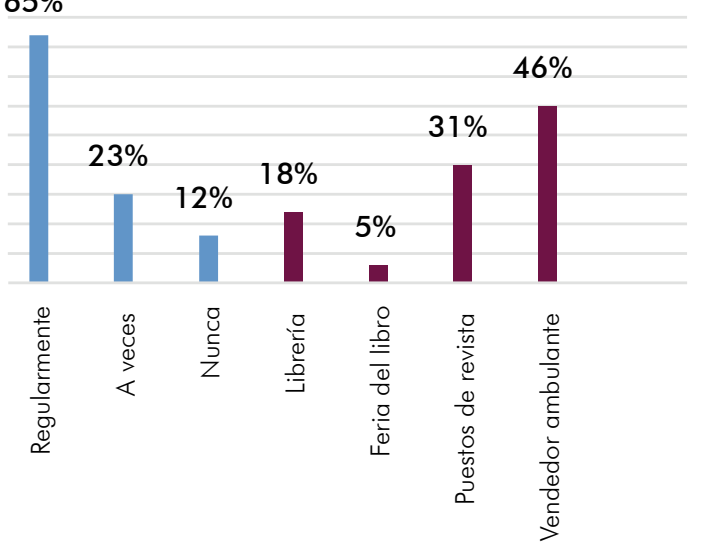

Nota. Resultados de la encuesta tipo de material de literatura infantil

En el cuarto y último apartado se habla si los padres de familia realizan con el niño actividades destinadas a estimular el hábito de la lectura como ir a la biblioteca, asistir a ferias de libro, entre otras; 13 padres (20\%) revelan realizar este tipo de actividades, en cambio 52 (80\%) afirman no realizarlas.

Lo que corresponde al conocimiento de algunas estrategias para estimular la lectura del niño/a; 56 progenitores de ambos niveles, que corresponden al $86 \%$ manifiestan no conocer estrategias y solo 9 (14\%) aseguran tener conocimiento del tema.
Figura 6

Actividades y estrategias para estimular la lectura

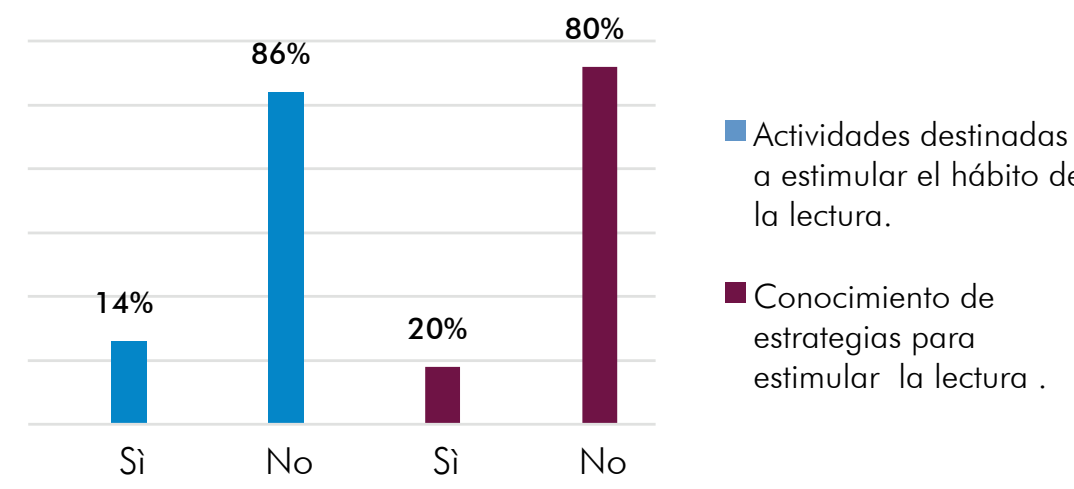

Nota. Resultados de la encuesta actividades y estrategias que emplean para estimular el hábito lector

\section{DISCUSIÓN}

La investigación tuvo como objetivo conocer las prácticas lectoras y las estrategias que los padres de familia utilizan para estimulan el hábito lector en sus hijos dentro del hogar, siendo su principal hallazgo la contradicción entre la importancia que afirman tener ambos grupos de padres de familia por la lectura frente al desconocimiento y la escasa inversión que dedican por la misma. Llama la atención que los padres de familia de ambos grupos responden a la encuesta afirmando que emplean la lectura únicamente para la realización de tareas escolares, antes que como medio de entretenimiento para sus hijos.

Este resultado constata que los padres de familia no poseen prácticas lectoras, deduciendo que no están dando la impor- 
tancia a estimular la lectura en casa y no ofrecen un modelo de aprendizaje para adquirir el hábito lector en sus hijos, debido a que el porcentaje obtenido que separa a la lectura escolar, por el entretenimiento familiar es mínimo, en otras palabras, nos da a entender que los padres están inculcando a la lectura más como una obligación académica que por placer. Situaciones similares a esta son reportadas por Santa (2017), los padres de familia que otorgan limitada importancia a la creación de ambientes familiares ricos en oportunidades para el aprendizaje y entrenamiento en lectura influyen negativamente en el posterior aprendizaje lector de sus hijos.

Además, la falta de un modelo de aprendizaje de la lectura en la familia puede ocasionar dificultades y bajos desempeños lectores en sus hijos, que causan un bajo rendimiento escolar. Dichos resultados son similares al estudio realizado por Bryant y Bradley (1983), donde los niños que no han crecido en un ambiente letrado, son más propensos a dificultades en la comprensión lectora.

En lo que respecta al apoyo e importancia a la lectura, los progenitores muestran simpatía hacia esta actividad, sin embargo, no se evidencian acciones afirmativas que respalden su respuesta, y esto se ve reflejado en el poco tiempo y el espacio que dedican a realizar actividades de lectura con sus hijos; contrastados con los resultados del INEC (2012), el tiempo dedicado sigue siendo un problema. Han pasado ocho años de esta última investigación y las estadísticas no presentan cambio alguno. Dándonos a entender que en los hogares no están dando a la lectura la debida valoración e importancia que requiere.
Por otra parte, los datos recogidos con la encuesta destacan que la mayoría de padres encuestados reportaron emplear Internet como primer recurso de lectura. Lo cual demuestra que las tecnologías de la información y comunicación están impactando en la adquisición del conocimiento e interacción social. Dicho aumento se debe al impacto que ha generado la pandemia del Covid-19 (Ciudades y Gobiernos Locales Unidos [CGLU], 2020).

Ahora bien, la mayoría de los padres solo posee un tipo de material de literatura infantil para estimular este hábito, y este no es adquirido de la manera correcta. Dado que la mayor parte de padres de familia compran este tipo de material a un vendedor ambulante y no en un lugar certificado como una librería (Escalante \& Caldera, 2008). Esto nos permite deducir que los padres compran este tipo de material a su gusto, sin tomar en cuenta las necesidades de sus hijos y las orientaciones pedagógicas.

En referencia a estrategias y actividades que aplican para estimular la lectura, en su gran mayoría afirman no tener conocimiento de estos aspectos, lo que produce una disonancia entre el gusto y la importancia que reportaron los padres y las acciones que realizan, sus respuestas estuvieron orientadas por la influencia de la deseabilidad social, antes que por el interés de ofrecer una figura sincera de relación con la lectura de sus hijos.

En síntesis, los resultados del presente trabajo permiten afirmar que los padres de familia muestran escasos conocimientos de cómo motivar el hábito lector de sus niños en el hogar, además se evidencian prácticas alfabetizadoras pasivas, dejando la responsabilidad a las instituciones educativas para instruir hábitos lectores en sus hijos, siendo poco su interés y esfuerzo en apoyar 
esta importante actividad. Estos resultados son nocivos para los niños que crecen en hogares donde no se estimula este hábito, en un futuro pueden presentar dificultades en el proceso de adquisición y comprensión de la lectura. Ahora bien, es importante tener presente que dichas afirmaciones se restringen al tamaño muestral del estudio, no pudiendo generalizarse a la población.

Con base en los resultados obtenidos de la investigación se permite sugerir la necesidad de involucrar a la familia en temáticas relacionadas al proceso de aprendizaje de la lectura, específicamente, en la enseñanza de estrategias y métodos que permitan construir hábitos lectores en sus hijos. Este tipo de iniciativas tienen la finalidad de mejorar la práctica lectora de los niños, la cual debe centrarse en promover la motivación de leer por placer, no solo en los pequeños, sino también en los adultos, pues ellos influyen en las costumbres que van adoptando los niños.

\section{CONCLUSIONES}

En esta investigación se resalta la importancia de fomentar el hábito de la lectura en la primera infancia, esta ayuda a que los niños sean autónomos y adquieran una capacidad reflexiva, logren mejorar la destreza de relacionar palabras y expresiones en los distintos contextos que le rodean, en especial el familiar, debido a que los pequeños toman como referente a los adultos que los rodean y estos se convierten en mediadores para su integración social y cultural.

Los padres de familia no han asumido el total compromiso de fomentar buenas prácticas lectoras en el hogar, dejando toda la responsabilidad formativa a la escuela, así lo reflejan los datos estadísticos de la investigación, en la que el $52 \%$ de los progenitores encuestados ven a la lectura más como una obligación académica que una actividad placentera, causando en los niños desinterés en la lectura.

Los padres de familia afirman que es importante la lectura, sin embargo, se evidenció que en la práctica la actividad lectora que realizan con sus hijos es mínima, considerando que no destinan un espacio físico adecuado para estimular este hábito y el tiempo empleado es de cinco a diez minutos cuando lo ideal, según los expertos, es de veinte a veinticinco minutos.

El material usado para la lectura de los niños en casa no es el adecuado, la investigación demostró que en los hogares se disponía como recurso literario en su gran mayoría solo de cuentos, el material debe ser variado, no solo los cuentos son un aliado para estimular la lectura, también existen otras alternativas como poesías, juegos de palabras, adivinanzas, fábulas, retahílas, canciones, rondas, entre otros. Estas composiciones literarias despiertan la curiosidad, creatividad e imaginación en los niños.

El $86 \%$ de los encuestados desconocen que existen actividades y estrategias para estimular correctamente el hábito de la lectura, cuyo propósito principal es brindar a los niños habilidades de comprensión y de agrado por la lectura para llegar a ser una persona lectora autónoma, crítica y de pensamiento creativo. Al mismo tiempo, dichos saberes deben partir de la cotidianidad de los infantes y de las experiencias comunicativas significativas. 
La limitación de este trabajo investigativo fue no contar con una mayor cantidad de población de padres de familia debido a la pandemia del Covid-19, lo que impidió que la muestra sea más significativa.

Futuras investigaciones deberán continuar actualizando, sistematizando resultados y profundizando en esta línea, particularmente sobre los factores que influyen en la adquisición de hábitos de lectura en los niños y las dificultades que traen consigo la falta de estimulación en el hogar, evidenciando el gran papel que cumple la familia en la adquisición y práctica de la lectura.

\section{AGRADECIMIENTOS}

Agradecemos a los padres de familia y a la directiva del comité Central Pro mejoras del barrio "San Francisco de Huarcay", por su gran acogida e interés en este trabajo y a Norma Parco, por su apoyo en la gestión de los respectivos permisos que requirió este estudio.

\section{REFERENCIAS}

Agencia Pública de Noticias del Ecuador y Suramérica ANDES. (2013). El índice de lectura del ecuatoriano es medio por año.: hHtp:// www.andes.info.ec/es/sociedad/indice-lectura-ecuatoriano-esmedio-libro ano.html

Baker, L., Scher, D., Baker, L., \& Scher, D. (2020). Influencias del hogar y la familia en las motivaciones para la lectura Influencias del hogar y la familia en las motivaciones para la lectura. 1-13.
Banihashemi, M., Rad, A. K., Yazdi, S. A. T., Rakhshande, H., Ghoyonlo, V. M., Zabihi, Z., \& Yousefzadeh, H. (2011). Evaluation of the effect of formic acid and sodium formate on hair reduction in rat. Clinical, Cosmetic and Investigational Dermatology, 4, 69-72. https://doi.org/10.2147/CCID.S20531

Bradley, L., \& Bryant, P. E. (1983). Categorizing sounds and learning to read: A causal connection. Nature, 301(5899), 419-421. https://doi.org/10.1038/301419a0

Bruner, S. J. (1984). Realidad mental y mundos posibles. Barcelona: Gedisa.

Bronfenbrenner, U. (1987). La ecología del desarrollo humano. Barcelona: Paidós.

Carrión Mieles, E., Zambrano Loor, T., \& Alarcón Barcia, L. (2017). Animación a la lectura y hábitos lectores en Centros de Educación Inicial de sectores Vulnerables. Revista Científica Sinapsis, 1 (10). https://doi.org/10.37117/s.v1i10.100

Centro Regional para el Fomento del Libro en América Latina y el Caribe (CERLALC). (2012). El espacio iberoamericano del libro. J. P. Mojica Gómez (Ed.) (pp. 132). http://www.cerlalc.org/files/ tabinterno/1d493d_Espacio_2012_digi_def.pdf

CGLU. (2020). Tecnologías digitales y la pandemia de COVID-19. Ciudades y Gobiernos Unidos, 17. https://www.uclg.org/sites/ default/files/eng_briefing_technology_es.pdf

Chávez Salgado, L. (2015). Estrategias para el fomento a la lectura: ideas y recomendaciones para la ejecución de talleres de animación lectora. E-Ciencias de La Información, 5(2), 1. https:// doi.org/10.15517/eci.v5i2.19605 
García, A., Ordoñez, L., \& Cusme Santander, C. (2016). Hábito de lectura en estudiantes de Centro de Educación, Manta provincia de Manabí. Dominio de Las Ciencias, 2(2), 280-290.

Educación, C. (2017.). Familias lectoras. Junta de Andalucía.

Escalante, D. T., Caldera, R. V. (2008). Disponible en: http://www. redalyc.org/articulo.oa?id $=35614570002$.

Ferreiro, D. (2000). Leer y escribir en un mundo cambiante 1. 1-8.

Hernández, R.; Fernández C. \& Batista, P. (2018) Metodología de la Investigación. (pp. 99-120) México. D.F: Mc-Graw-Hill.

Howie, S., Combrinck, C., Tshele, M., Roux, K., Palane, N., \& Mokoena, G. (2017). Pirls 2016: South African Children's Reading Literacy Achievement.

INEC. (2012). Hábitos de lectura en Ecuador. Instituto Nacional de Estadística y Censos.

Kalb, G., \& Ours, J. C. Van. (2020). Leyendo a niños pequeños: ¿̇Una ventaja en la vida? 7416, 1-36.

Maluf, M. R., \& Sargiani, R. de A. (2013). Lo que la neurociencia tiene que decir sobre el aprendizaje de la lectura. Revista de Psicología de Arequipa, 3(1), 11-24.

Mata, J. (2016). Neurociencia, lectura y literatura infantil. October, $48-51$.

Matvichuk, T. (2020). La influencia de las expectativas de los padres, el hogar Ambiente de alfabetización y comportamiento de los padres en el Interés de lectura infantil. 1-43.

Milton, V. (2010). Confiabilidad y coeficiente Alpha de Cronbach Licenciado Educación Mención Matemática y Física. Profesor de Educación Integral. Revista de Estudios Interdisciplinarios En Ciencias Sociales, 12(2), 248-252. https://doi.org/10.1007/ s00259-006-0152-0

Ministerio, E, C. (2016). Familias lectoras: cómo formar lectores y escritores en el hogar http://aprende.colombiaaprende.edu.co/ sites/default/files/naspublic/familias_lectoras.pdf.

Ministerio, E, E. (2019). Sin lectura no hay educación. La lectura en familia, 10-24. https://educacion.gob.ec/wp-content/uploads/ downloads/2019/1 1/guia-metodologica-para-desarrollar-elgusto-por-la-lectura.pdf

Moreno, E. (2001). Análisis de la influencia de la familia en los hábitos lectores de sus hijas e hijos: Un estudio etnográfico. Contextos Educativos. Revista de Educación, 0(4), 177. https://doi. org/10.18172/con.492

Nádas, J., \& Rakovics, V. (2017). High intensity broad spectrum LEDs in the near infrared range. Materials Science Forum, 885, 141-146. https://doi.org/10.4028/www.scientific.net/MSF.885.141

OCDE. (2019). Informe PISA 2018. https://doi.org/08887543(95)80088-4 [pii]

Ortiz, M. (2017). La importancia del hábito por la lectura en niños de primaria menor. Glosa Revista de Divulgación, 5(9), 244-260.

Paredes M., J. (2014). La escuela y el desafío del hábito de la lectura. Razón y Palabra, 89, 31-76.

Ramírez, C., \& De Castro, D. (2013). La lectura en la primera infancia. Grafías Disciplinares de La UCP, 20, 7-21. https://dialnet.unirioja. es/servlet/revista? codigo $=15759$

Rendón, V. (2018). Fortalecimiento de la lectura mediante la interacción familiar: programa PROLECS-UNA. Educere, 20(66), 299-309. 
Roque, L., \& Álvarez, C. (2016). Estimulación temprana de la lectura desde la Educación Infantil. Lenguaje y Textos, 43, 123. https:// doi.org/10.4995/lyt.2016.5942

Rosero, A. L., Mieles, M. D. (2015). Familia y lectura en la primera infancia: una estrategia para potenciar el desarrollo comunicativo, afectivo, ético y creativo de los niños y niñas. Itinerario Educativo, 29(66), 205. https://doi.org/10.21500/01212753.2220

Santana Hernández, R., Alemán Falcón, J. A., \& López Torrijo, M. (2017). Leer por placer: ilo primero, oír los libros! Aula Abierta, 46, 83. https://doi.org/10.17811/rifie.46.2017.83-90

Sepúlveda Velásquez, L., \& Gaintza Jauregui, Z. (2017). Perspectivas inherentes al desarrollo del lenguaje. Aportaciones desde la psicolingüística. Paulo Freire, 13, 65. https://doi. org/10.25074/07195532.13.411

Tamayo, T. M. (2019). Revista Andina de Educación El sistema educativo de Ecuador: un sistema, dos mundos. 2(1), 8-17.

UNESCO (2016), "TERCE: aportes para la enseñanza de la lectura", Santiago de Chile, OREALC/ UNESCO, en: http://unesdoc. unesco.org/images/0024/002448/244874S.pdf

Vasylenko, O. V. (2020). El papel de los padres en ayudar a los niños a desarrollar habilidades de lectura Rola. 1-10.

Yusof, N. M. (2020). Influencia de los factores familiares en los hábitos de lectura e interés entre alumnos de nivel 2 en escuelas primarias nacionales en Malasia. 5(2010), 1160-1165. 


\section{BIOGRAFÍA DE LOS AUTORES}

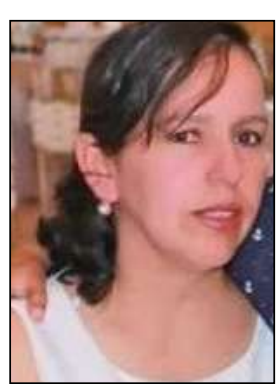

ROSARIO REQUENA V.

Docente investigadora UTPL. Licenciada en Secretariado Ejecutivo, Magíster en Educación a Distancia - UTPL. Máster Universitario en Didáctica de la Lengua y la Literatura en Educación Secundaria y Bachillerato (UNIR). Docente universitaria de Didáctica Específica de la Lengua y Literatura, Introducción a la Lingüística, Lingüística Contemporánea, Lectura y Redacción de Textos, entre otras.

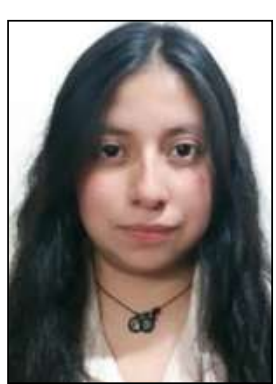

\section{EVELYN SAGAL}

Estudiante de la Carrera de Educación Inicial en la Universidad de las Fuerzas Armadas ESPE. Con experiencia laboral como auxiliar de Inicial en las instituciones: Centro de Desarrollo Infantil "Happy Children" y Escuela fiscal "Roberto Cruz". Inclinación investigativa en temas relacionados con el desarrollo del lenguaje en niños pequeños y la enseñanza de la lectura en base a la neurociencia.

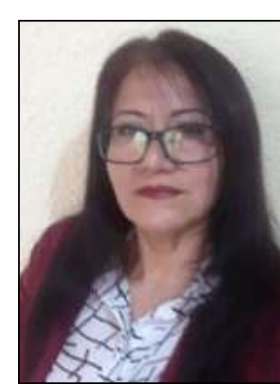

\section{VERÓNICA CARVAJAL}

Docente titular Universidad de las Fuerzas Armadas ESPE. 25 años experiencia. Formación: Licenciatura en Educación Parvularia, Doctorado 3er nivel en Investigación Planificación Educativa, Maestría Intervención Dificultades del Aprendizaje Cursos de especialidad en Neuropsicología, Neuroeducación, Integración Sensorial, Educación Inclusiva. Cargos: Equipo Rediseño, Acreditación DCHS, Coordinadora área Psicología. 